\title{
Ion-Assisted Deposition of Amorphous PbO Layers
}

\author{
O. Semeniuk ${ }^{1,2 *}$, A. Csik ${ }^{3}$, S. Kökényesi ${ }^{4}$, A. Reznik ${ }^{2,5}$
}

1 Chemistry and materials science program, Lakehead University, 955 Oliver Road, Thunder Bay, ON, Canada P7B 5E1

2 Advanced detection devices department, Thunder Bay Regional Health Research Institute, 290 Munro Street, Thunder Bay, ON Canada P7A 7T1

3 Institute for Nuclear Research, Hungarian Academy of Sciences, Bem tér 18/c, P.O. Box 51, H-4001, Debrecen, Hungary

4 Institute of Physics, University of Debrecen, H-4026, Bem sq.18/a, Debrecen, Hungary

5 Department of Physics, Lakehead University, 955 Oliver Road, Thunder Bay, ON, Canada P7B 5E1

*Corresponding author.

E-mail address: osemeniu@lakeheadu.ca (O. Semeniuk).

\begin{abstract}
Lead oxide $(\mathrm{PbO})$ is one of the most promising materials for application in direct conversion medical imaging $\mathrm{X}$-ray detectors. Despite its high potential, conventional polycrystalline $\mathrm{PbO}$ layers deposited with the basic thermal evaporation method are not yet mature for practical use in x-ray imaging; indeed, they are highly porous, unstable at ambient conditions, and sub-stoichiometric. In order to combat the above issues with $\mathrm{PbO}$, we advance the basic evaporation process with simultaneous energetic ion bombardment of the growing film. We show that tuning the ion-assisted thermal deposition not only solves the structural problems of poly- $\mathrm{PbO}$, but also enables the growth of a new non-crystalline polymorphic form of the material - amorphous $\mathrm{PbO}$ (a-PbO). In contrast to poly- $\mathrm{PbO}$, novel a$\mathrm{PbO}$ layers grown by ion-assisted thermal deposition are stable at ambient conditions. Structural and morphological analysis confirms that a-PbO is stoichiometric and free of detectable voids, which suggests higher bulk X-ray stopping power than porous poly- $\mathrm{PbO}$.
\end{abstract}

Keywords: Oxide-film growth kinetics, phase transformation, polycrystals, amorphous. 


\section{Introduction}

There is great interest in the utilization of non-crystalline photoconductors for direct conversion medical X-ray imaging detectors. Lead Oxide $(\mathrm{PbO})$ is one of the most promising candidates for this purpose, since it has high stopping power due to high atomic number of $\mathrm{Pb}$ and has high theoretical $\mathrm{X}$-ray-to-charge conversion gain. In addition, poly- $\mathrm{PbO}$ room temperature deposition technology allows the deposition of a thick photoconductive layer directly on any imaging electronics, thus permitting a custom $\mathrm{PbO}$ detector design to fit the requirements of a variety of clinical applications.

Conventional poly- $\mathrm{PbO}$ layers are deposited by basic thermal evaporation in the atmosphere of molecular oxygen from a crucible loaded with high purity $\mathrm{PbO}$ powder and heated above $\mathrm{PbO}$ melting temperature $T_{\text {melt }} \sim 890$ ${ }^{\circ} \mathrm{C}$ [1]. For use in the direct conversion $\mathrm{x}$-ray detectors, the $\mathrm{PbO}$ layer has to be deposited on a substrate containing

image read-out electronics [2]. In this case the substrate temperature is maintained at $T_{\text {sub }} \sim 100{ }^{\circ} \mathrm{C}$ as dictated by thermal tolerance of the majority of commercial read-out electronics [3]. The large mismatch between the substrate temperatures $T_{\text {sub }}$ and the melting temperature of $\mathrm{PbO} T_{\text {melt }}$ results in low packing density and high porosity of grown layers $[4,5]$ : the density of as-grown poly- $\mathrm{PbO}$ layers is much lower than that of a crystalline material (up to $50 \%$ of single crystal density), which significantly decreases the X-ray attenuation of the grown film. A typical poly- $\mathrm{PbO}$ layer is composed of randomly oriented platelets about one micron in diameter and one hundred nanometers thick and exhibits a rough surface morphology $[1,6]$. The grown PbO films are known to consist of two different crystallographic phases of $\mathrm{PbO}$ : the seeding layer, several microns thick, is formed by the yellow orthorhombic $\beta-\mathrm{PbO}$, while the bulk of the layer grows predominately as a red tetragonal lead oxide $(\alpha-\mathrm{PbO})$, however, $\beta$ - $\mathrm{PbO}$ is also present in the bulk [7]. In addition, the grown layers are substoichiometric with a deficit of oxygen [8-10]. The latter is due to the low formation energy of oxygen vacancies $[10,11]$ which can act as charge trapping centers - a highly undesirable feature for any detector material. Poly-PbO layers are unstable in air and transform into Hyrdo Cerrusite under ambient conditions [12].

Suboptimal packing density, poor sample stability and oxygen deficiency are well known problems of many oxides deposited with basic thermal or electron beam evaporation techniques. These issues were successfully combated by means of simultaneous bombardment of growing film with energetic ions. Here, a practical and easy to implement way of obtaining energetic ions is by utilization of a specially designed ion source during the deposition process $[4,13]$. 
The ion assisted depositions have proven to be a versatile tool for solving a broad range of material science challenges. Initially this method was employed to improve the basic properties of metal oxides, including packing density [14-17] and stoichiometry [18,19]. Nowadays, ion assisted deposition is successfully applied to advance the growth of organic transistors and polymers-based electronic devices [20-22]. In addition, ion assisted deposition is used as an alternative to high pressure and/or high temperature deposition technique of crystalline and epitaxial materials $[23,24]$.

Motivated by the success of ion-assisted deposition to improve structure and stability of other materials, we applied this technique to $\mathrm{PbO}$ layers growth. The structural characterization performed with Raman spectroscopy, X-ray diffraction (XRD) and X-ray Photoelectron Spectroscopy (XPS), and morphological examinations with Scanning Electron Microscopy (SEM) revealed a new polymorphic form of $\mathrm{PbO}$ - an amorphous (a-PbO) material that appeared to be dense and stable at ambient conditions. a-PbO samples are free of voids, possess bulk packing density, and have perfect stoichiometry throughout the film thickness as will be shown below.

\section{Materials and Methods}

Polycrystalline $\mathrm{PbO}$ (poly-PbO) sample preparation

Poly- $\mathrm{PbO}$ layers were prepared by conventional thermal evaporation of $\mathrm{PbO}$ powder as shown in Fig. 1 (a). A $\mathrm{Pt}$ crucible, containing pure $(5 \mathrm{~N}) \mathrm{PbO}$ powder obtained commercially from Chemsavers, is loaded into the furnace (evaporation source) of the vacuum chamber. Typically, the evaporation takes place at temperature $\sim 1000{ }^{\circ} \mathrm{C}$ which provides a growth rate $\sim 1 \mu \mathrm{m} / \mathrm{min}$. The evaporated particles of $\mathrm{PbO}$ (vapor stream) condense on the rotating substrate that is kept at $\sim 100^{\circ} \mathrm{C}$. In order to improve the layer stoichiometry, the deposition takes place under $\sim 0.2$ Pa background pressure of high purity molecular oxygen, which is directly supplied to the vacuum chamber $[1,11]$.

\section{Amorphous $\mathrm{PbO}(\mathrm{a}-\mathrm{PbO})$ sample preparation}

$\mathrm{a}-\mathrm{PbO}$ layers were prepared by thermal evaporation with low energy oxygen ion bombardment. The key difference from the conventional deposition described above, is that the deposition takes place in the atmosphere of ionized oxygen, instead of molecular oxygen. This is achieved by running molecular oxygen through an ion source placed at $\sim 10 \mathrm{~cm}$ below the substrate holder with a beam incidence angle of $\sim 45^{\circ}$ to the normal of the substrate as shown in Fig. 1 (b). In our system the ion source utilizes a hot filament to produce electrons. Electrons are 
accelerated by the applied electric field which is towards the anode of the ion source were they bombard $\mathrm{O}_{2}$ introduced into the ion source body, producing oxygen ions [18]. The ion source allows for independent control of oxygen ion energy and ion flux (ion current density), required to achieve the desired structural modifications of a growing layer. Thus, during the typical ion assisted deposition, the ion energy was between $40-60 \mathrm{eV}$, while the ion flux was $\sim 0.2 \mathrm{~mA} / \mathrm{cm}^{2}$. The substrate and evaporation source temperatures were similar to those of the conventional thermal evaporation, resulting in the deposition rate of a- $\mathrm{PbO} \sim 0.4 \mu \mathrm{m} / \mathrm{min}$.

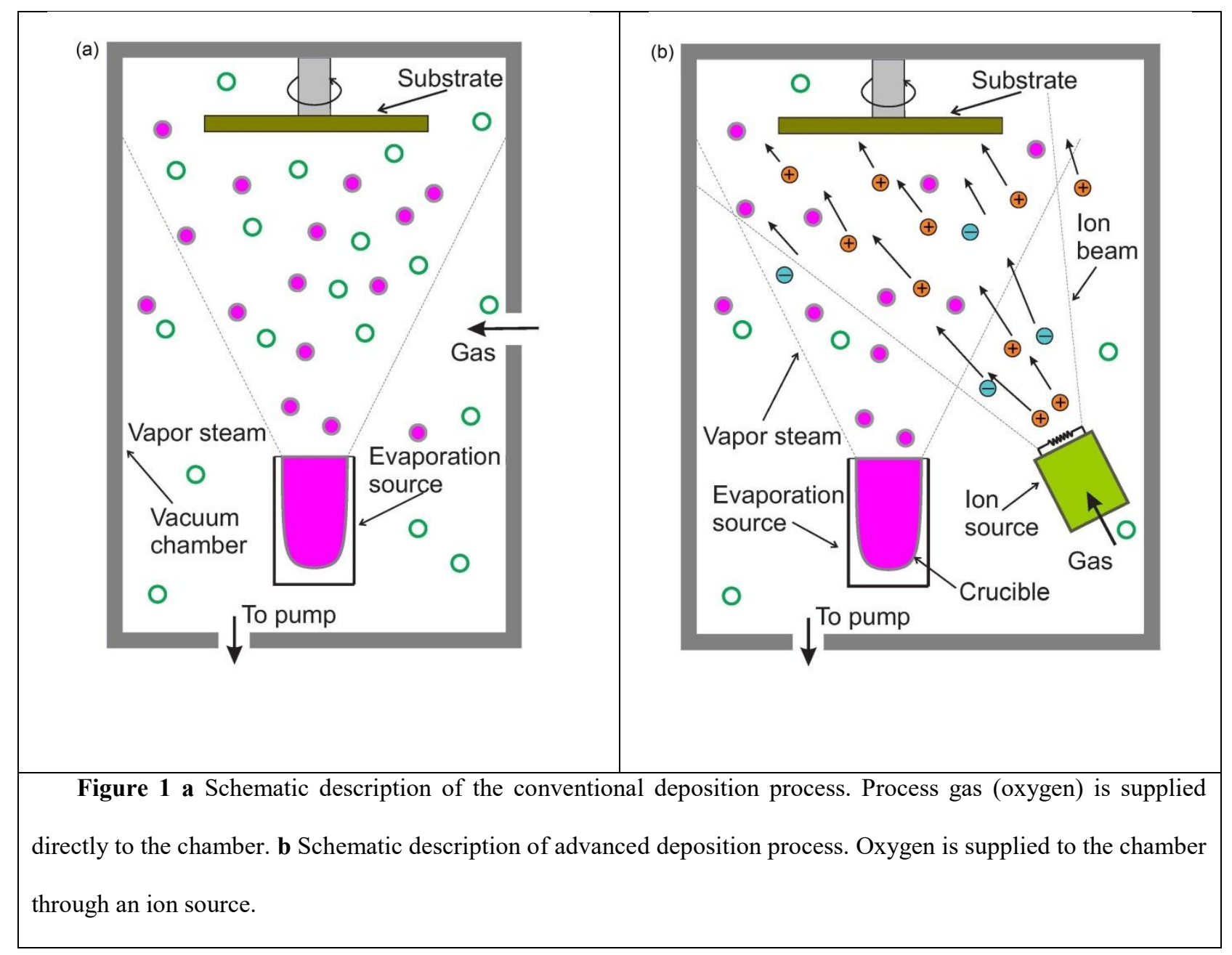

\section{Sample characterization}

The structural and morphological analyses of poly- and a-PbO samples were performed with Raman, X-Ray diffraction (XRD) and X-ray Photoelectron Spectroscopy (XPS) and Scanning Electron Microscopy (SEM).

Since poly- $\mathrm{PbO}$ samples are known to transform into Hydro Cerrisute under ambient conditions, they were stored under the protective dry nitrogen atmosphere. They were exposed to ambient only for the time of the Raman 
spectrum acquisition and when the sample was relocated from one measurement system to the other. This maximal exposure time (during Raman spectroscopy) did not exceed 10 minutes, therefore no structural changes were observed. During XRD measurement, the samples were under continuous argon gas purge. XPS measurement was done in high vacuum conditions (base pressure $\sim 5 \times 10^{-6} \mathrm{~Pa}$ ). One poly-PbO sample was stored under ambient conditions and was used as a reference. In order to establish stability of novel a-PbO layers, they were stored under ambient conditions.

\section{Raman and XRD spectroscopies.}

Raman spectroscopy measurements were performed with a Renishaw inVia Raman spectrometer with resolution of $\sim 1 \mathrm{~cm}^{-1}$. The Raman spectra were measured with an Ar ion laser, using the $785 \mathrm{~nm}$ laser line at 1200 $\mathrm{lp} / \mathrm{mm}$ mesh. Prior to the measurements, the system was calibrated to silicon peak at $520 \mathrm{~cm}^{-1}$. The intensity level was carefully adjusted in order to prevent light induced changes, which was especially important for a-PbO samples.

XRD spectroscopy was performed with a Pananalytical Expert Pro Diffractometer and the X-ray source was $\mathrm{CuK} \alpha$ radiation with wavelength $0.15405 \mathrm{~nm}$. The typical X-ray diffraction pattern was recorded from $2 \theta=20^{\circ}$ to $90^{\circ}$ with the scan step size of $0.01313^{\circ}$. The phase identification was achieved by the comparison with data from the JCPDS International diffraction data base.

For a-PbO samples, Raman and XRD measurements were performed on both, as-grown layers and layers undergoing one hour annealing in a temperature range from 200 to $600{ }^{\circ} \mathrm{C}$. After annealing at each temperature, the sample was slowly cooled down with the rate of $\sim 1{ }^{\circ} \mathrm{C} / \mathrm{min}$ in order to prevent thermal shock. After the XRD and Raman measurements were performed on the cooled sample, it was moved back to the oven for the annealing at a subsequent temperature. The upper temperature limit was dictated by thermal stability of the glass substrate. The annealing took place in the argon atmosphere in order to prevent over-oxidation of the samples.

\section{XPS spectroscopy}

Stoichiometry of poly-and amorphous $\mathrm{PbO}$ layers have been investigated by X-Ray Photoelectron Spectroscopy (XPS). The XPS spectra of PbO layers were investigated with a PHOIBOS 100 analyzer (SPECS GmbH, Berlin) equipped with Al X-Ray source. The NIST XPS Database [25] was used for peak identification and CasaXPS 
software was used for deconvolution and fitting of $\mathrm{Pb} 4 \mathrm{f}$ and O1s peaks. Carbon $(\mathrm{C} 1 \mathrm{~s})$ peak with binding energy of $284.5 \mathrm{eV}$ was used as a reference for calibration of energy.

In order to investigate any changes in-depth of the sample, the tested films were sputtered in-situ in a Secondary Neutral Mass Spectrometry (SNMS) [26] chamber connected to the XPS. Thus, the first XPS spectrum was recorded on the original surface (as-prepared) of the sample and afterwards the sample was moved into the SNMS chamber (base pressure $0.2 \mathrm{mPa}$ ) for sputtering with $\mathrm{Ar}^{+}$ion bombardment. After each sputtering the new XPS spectrum was recorded and the sample was moved back into the SNMS chamber for the next sputtering process. The lateral homogeneity of ion bombardment was checked by measuring the shape of the sputtered crater at the end of the experiment with an AMBIOS XP-1 type profilometer. A profilometer was also used to determine the sputtering rate by measuring both the crater depth and sputtering time. The poly-PbO sample was sputtered for 120, 420, 1020 and 1620 seconds, which correspond to 140, 510, 1250 and $1950 \mathrm{~nm}$ sputter depths, respectively. Similarly, a-PbO was sputtered for 60, 360, 960 and 1860 seconds, corresponding to 15, 87, 232 and $450 \mathrm{~nm}$ sputter depths.

Due to the high resistivity of $\mathrm{PbO}$ samples, a large charge accumulation effect occurred on the sputtered surface as a result of $\mathrm{Ar}+$ ion bombardment during the sputtering process. In order to account for this sample charging effect the SNMS was operated in the High Frequency (HF) operation mode (at $100 \mathrm{kHz}$ with $50 \%$ duty cycle), when a square-wave type high frequency voltage is applied to the sample instead of a constant DC voltage $[27,28]$. This operation mode allows neutralization of the positive charge accumulated on the surface during ion bombardment periods.

\section{Results}

\section{Morphological analysis}

A SEM micrograph of the surface of the poly- $\mathrm{PbO}$ prepared with conventional deposition method, is shown in Fig. 2 (a). As seen from Fig. 2 (a), poly- PbO film consists of a network of platelets, each less than 2 microns in diameter and about $50 \mathrm{~nm}$ thick. On the film surface, platelets are seen to be oriented primarily in the growth direction (although arbitrary relative to each other). This platelet structure is highly porous and results in high surface roughness, which is on the range of a micrometer, as defined by the size of the platelets. It should be noted that the morphology of poly-PbO layers grown here is very similar to that reported previously by Simon et al. [1] suggesting high reproducibility of the basic thermal evaporation process. 
Fig. 2 (b) shows the SEM picture of the amorphous $\mathrm{PbO}$. The film is seen to be uniform, free from voids and does not exhibit a platelet structure typical of poly- $\mathrm{PbO}$. The absence of a platelet network results in a smooth surface of the grown layer, never achieved before with polycrystalline films.
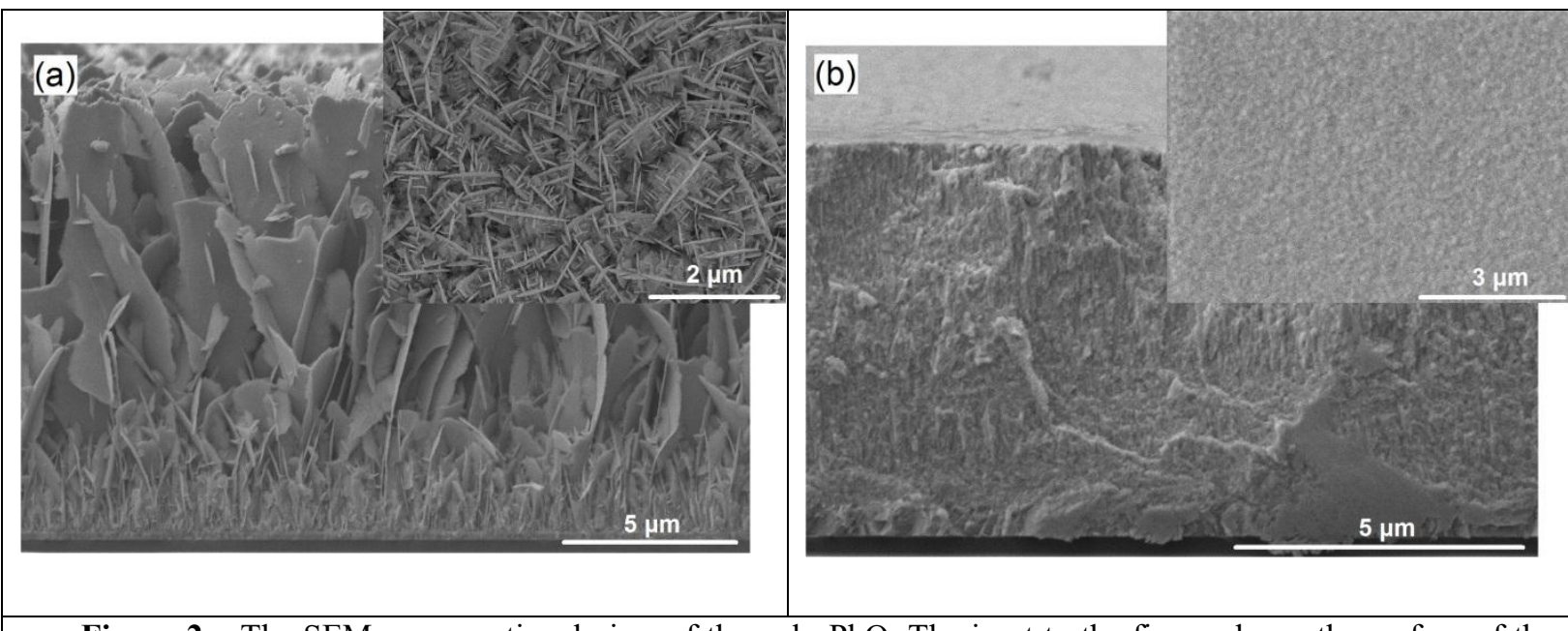

Figure 2 a The SEM cross-sectional view of the poly- $\mathrm{PbO}$. The inset to the figure shows the surface of the poly- $\mathrm{PbO}$ layer. b SEM cross-sectional view of the a-PbO. The inset to the figure shows the surface of the a-PbO layer.

\section{Raman spectroscopy}

Fig. 3 (a) compares Raman spectra of as-deposited poly- and a-PbO films. The Raman spectrum of poly-PbO indicates the presence of both phases: tetragonal $\alpha-\mathrm{PbO}$ peaks at 81 and $340 \mathrm{~cm}^{-1}$ and $\beta-\mathrm{PbO}$ peaks at 89 and 289 $\mathrm{cm}^{-1}$. The Raman peak at $\sim 146 \mathrm{~cm}^{-1}$ cannot be unambiguously attributed to either phase, since both of them have the strongest signal in this region: $\alpha-\mathrm{PbO}$ at $145 \mathrm{~cm}^{-1}$ and $\beta-\mathrm{PbO}$ at $147 \mathrm{~cm}^{-1}$.

Since the poly- $\mathrm{PbO}$ sample was stored under protective atmosphere of dry nitrogen, no Hydro Cerrisute peaks were detected. However, another poly-PbO sample from the same deposition run which was kept at ambient conditions developed a Hydro Cerrisute peak at $1050 \mathrm{~cm}^{-1}$ on the Raman spectrum.

Overall, the measured Raman spectrum of poly- $\mathrm{PbO}$ is represented by high and relatively narrow peaks, indicating crystalline structure of the deposited layers. In contrast, Raman spectrum of novel $\mathrm{PbO}$ is very different. It is represented by a wide peaks and a high plateau typical to amorphous structure. 


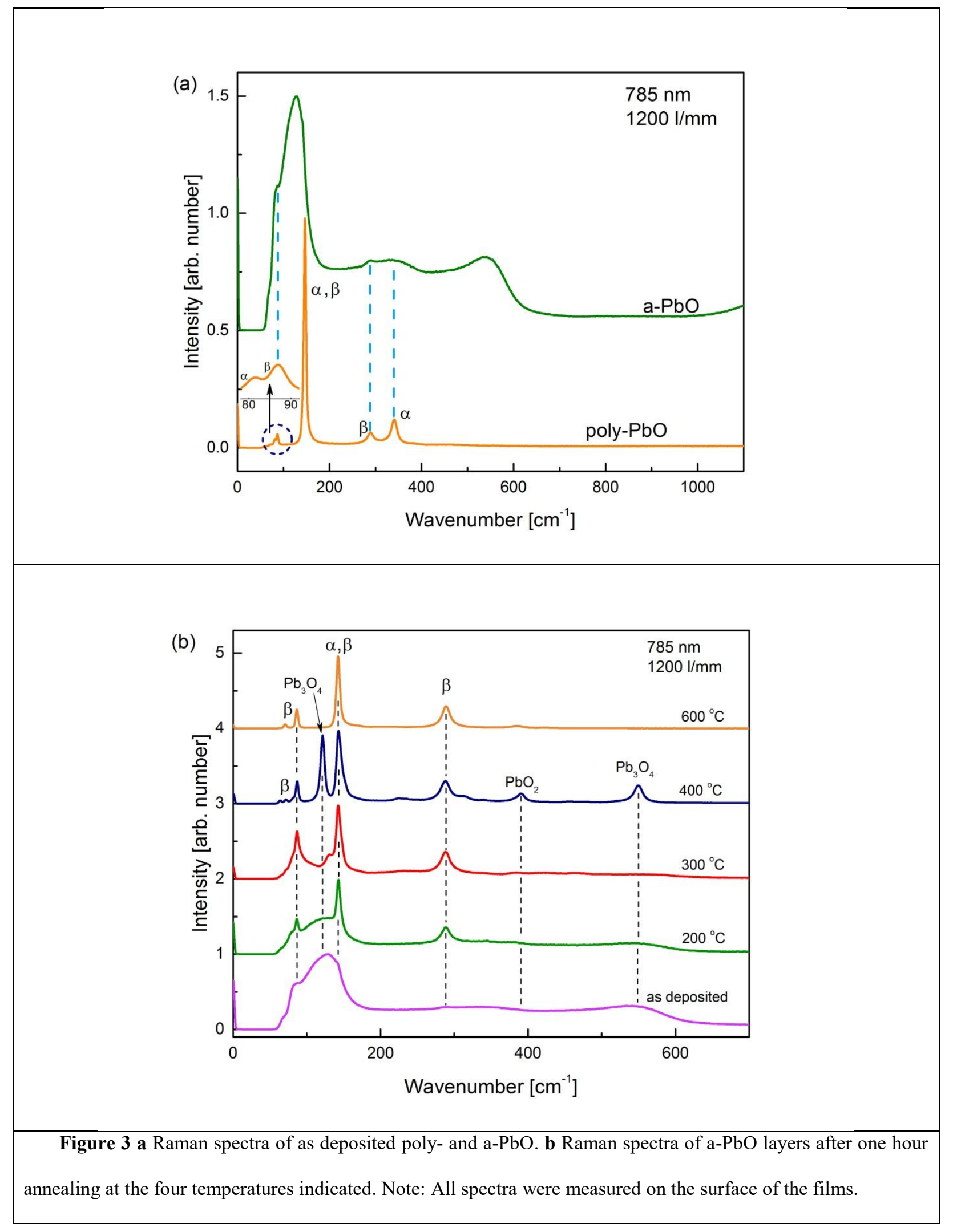


Fig. 3 (b) shows gradual changes in Raman spectra of a-PbO following one hour annealing at four different annealing temperatures. As is clear from Fig. 3 (b), the a-PbO layer undergoes gradual re-crystallization, evident from the characteristic peaks which evolve from a broad spectrum. At $200{ }^{\circ} \mathrm{C}$, crystallization begins with the formation of the peaks at 85,143 and $288 \mathrm{~cm}^{-1}$; in the temperature range from $200{ }^{\circ} \mathrm{C}$ to $300{ }^{\circ} \mathrm{C}$ these peaks grow gradually and become well defined at $300{ }^{\circ} \mathrm{C}$. These peaks are close to those of the $\beta$-PbO phase [7]. At $400{ }^{\circ} \mathrm{C}$, another characteristic $\beta$-PbO peak appears at $71 \mathrm{~cm}^{-1}$, as well as additional peak at $121,224,391$ and $548 \mathrm{~cm}^{-1}$ attributed to $\mathrm{Pb}_{3} \mathrm{O}_{4}$ [7]. Upon annealing to $600{ }^{\circ} \mathrm{C}$, most of the former peaks have disappeared. All peaks at $600{ }^{\circ} \mathrm{C}$ are attributed to orthorhombic $\mathrm{PbO}$. The spectra showed in Fig. 3 were normalized and shifted upwards for better visualization, however it is worth mentioning, that with higher temperature the peaks become taller and narrower. At the same time plateaus decrease with increasing temperature, indicating refinement of the crystal structure.

It should be noted that a-PbO samples did not require a special protective environment. Thus, the Raman spectra of the a-PbO samples stored at ambient conditions persisted over time and did not show any features of Hydro Cerrusite.

\section{XRD spectroscopy}

The XRD spectra of as-grown poly- and a-PbO films are shown in Fig. 4 (a). While the XRD spectrum of poly$\mathrm{PbO}$ is represented by sharp, well defined characteristic peaks, indicative of crystalline ordered structure, the XRD of a-PbO does not exhibit distinct narrow peaks indicating its amorphous nature.

$\mathrm{X}$-Ray diffraction measurements of the poly- $\mathrm{PbO}$ sample reveal the presence of both tetragonal and orthorhombic phase of $\mathrm{PbO}$, as well as an orthorhombic $\mathrm{PbO}_{2}$ phase, which was not observed on Raman spectrum. This might be due to the fact that the sensitivity of the XRD measurements is higher than that of Raman spectroscopy. As for the a-PbO layer, its amorphous structure changes dramatically upon annealing (Fig. 4 (b)). The effect of annealing starts to appear at $200{ }^{\circ} \mathrm{C}$, although very insignificantly. At higher temperatures, characteristic peaks emerge from a broad spectrum. A gradual refinement of the crystal structure is further observed: width of the emerged peaks decreases and their intensity increases with increasing temperature. At temperatures above of $300{ }^{\circ} \mathrm{C}$, multiple phases of $\mathrm{PbO}$ were found to coexist simultaneously: $\alpha, \beta-\mathrm{PbO}, \mathrm{Pb}_{3} \mathrm{O}_{4}$ and $\mathrm{PbO}_{2}$. However at $600{ }^{\circ} \mathrm{C}$, the spectrum closely resembling a single phase of orthorhombic $\mathrm{PbO}$ with a minor peak of $\mathrm{PbO}_{2}$ detected. 

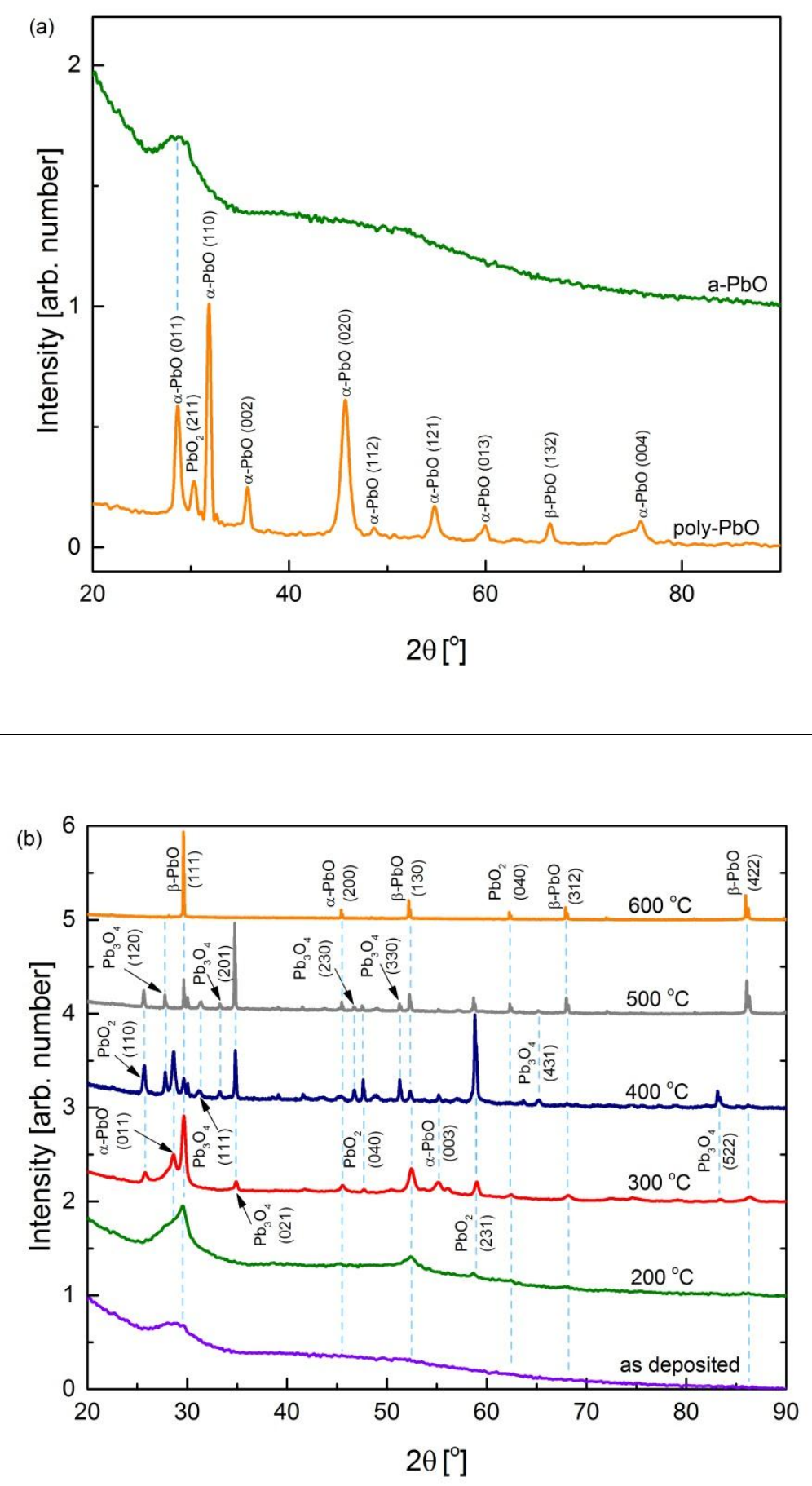

Figure 4 a The XRD spectra of as-grown poly- and a-PbO films. b The XRD spectra of as deposited amorphous $\mathrm{PbO}$ layer as well as the same layer annealed at five different temperatures for one hour. The spectra were measured on the surface of the films. 


\section{XPS spectroscopy}

Fig. 5 (a) shows the XPS spectra of the poly-PbO sample measured on the surface and in the bulk of the sample. The surface measurements indicate the presence of both $\mathrm{PbO}$ and $\mathrm{PbO}_{2}$ phases. The $\mathrm{PbO}_{2}$ peak disappears when measurements are taken at $140 \mathrm{~nm}$ in depth, however, a small amount of metal $\mathrm{Pb}$ phase is seen to be present in the spectrum. Spectra measured at a depth of $510 \mathrm{~nm}$ closely resemble that of $140 \mathrm{~nm}$. However, with further sputtering, an additional contribution of pure $\mathrm{PbO}_{2}$ appears in the measurements, while $\mathrm{Pb}-\mathrm{O}$ remains the dominant bonding configuration.

Fig. 5 (b) shows the XPS spectra of a-PbO measured on the surface and after sputtering in SNMS system. As can be seen from the presented spectra, the composition of the film remains constant across the layer thickness: only lead-oxide $(\mathrm{PbO})$ without any other elements or oxides of lead (e.g. $\left.\mathrm{PbO}_{2}\right)$ was detected.
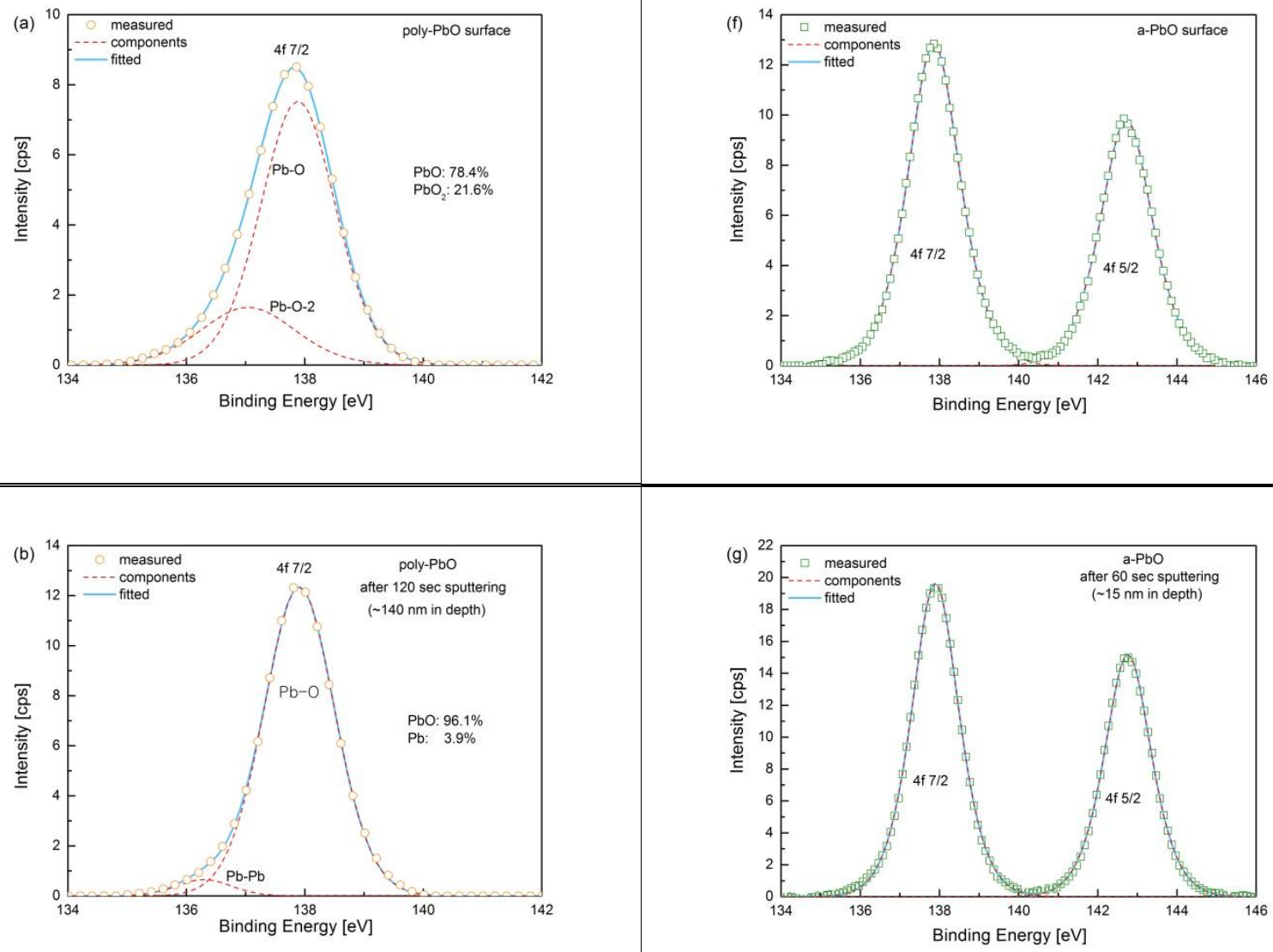

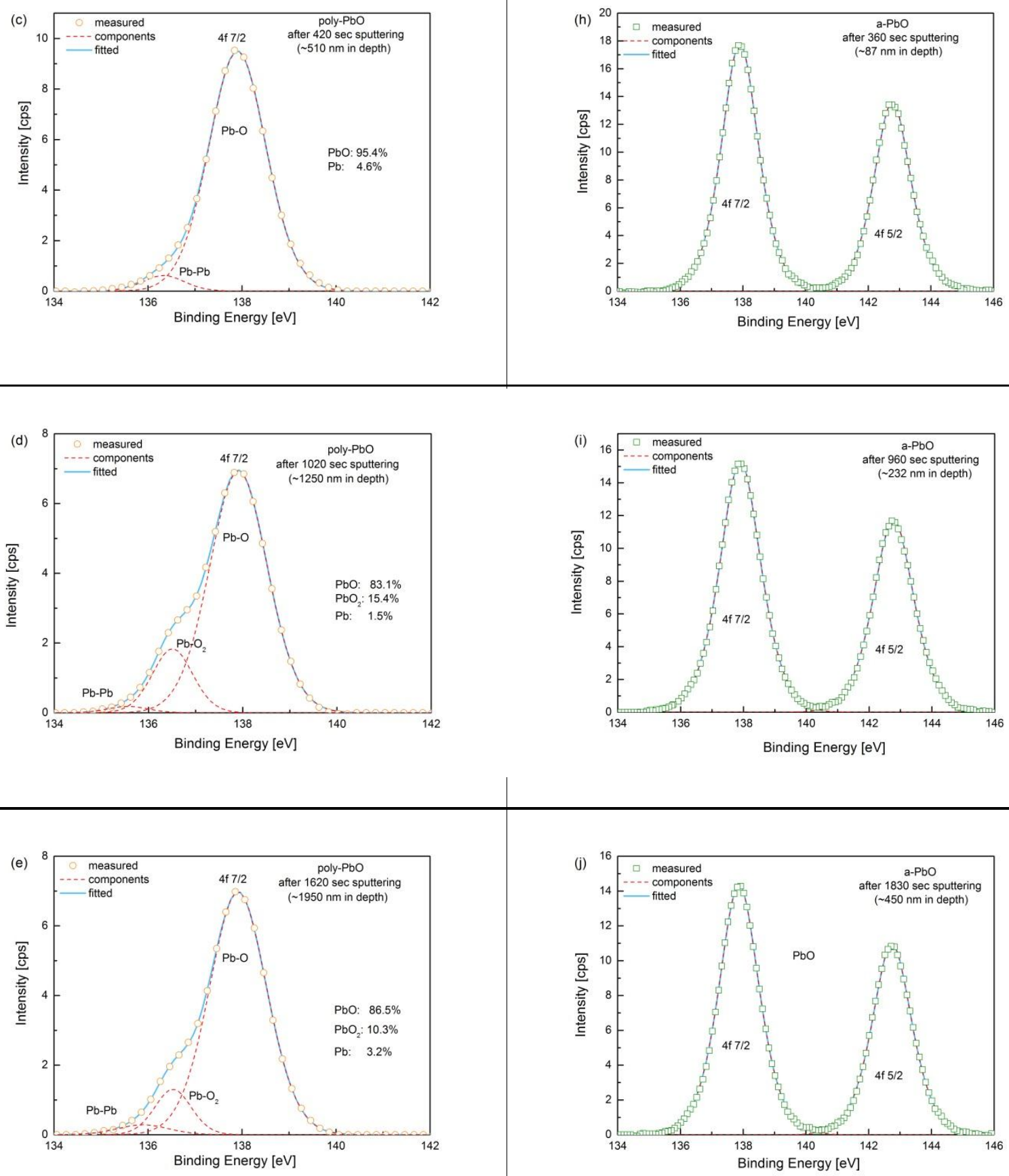

Figure 5 a-e XPS spectra of poly- $\mathrm{PbO}$ and $\mathbf{f}-\mathbf{g}$ a-PbO at selected depths.

\section{Discussion}

Morphological and structural properties of $\mathrm{PbO}$ layers deposited with both basic thermal evaporation and ionassisted thermal evaporation were investigated with multiple characterization techniques, including SEM, Raman 
spectroscopy, XRD and XPS. While Raman and XRD measurements were taken on the layers' surfaces, XPS spectra were measured at different depths, monitoring stoichiometry and composition across the layers.

Qualitatively, all the measurements taken confirm that while basic thermal evaporation results in polycrystalline structure of the grown layers, oxygen ion bombardment with optimized ion energy and flow results in a new polymorphic form of the grown material, namely, amorphous Lead Oxide (a-PbO) not synthesized before. In contrast to the poly- $\mathrm{PbO}, \mathrm{a}-\mathrm{PbO}$ is uniform, free of voids and does not require special storage conditions to prevent structural degradation. In addition, morphological analysis of $\mathrm{PbO}$ layers indicates that novel a-PbO layers possess a significantly higher packing density, in comparison with poly-PbO (see Fig. 2). Improved density and absence of voids in the layer offers a high bulk X-ray stopping power - one of the main advantages of $\mathrm{PbO}$ for direct conversion X-ray detectors.

Although amorphous $\mathrm{PbO}$ is stable at ambient conditions, it is (as expected) fundamentally metastable with respect to the crystalline structure and undergoes gradual crystallization toward orthorhombic $\mathrm{PbO}$ at elevated temperatures as shown with XRD and Raman measurements after different stages of thermal annealing. This property is reminiscent of another amorphous material which is highly significant to radiation medical imaging, namely, amorphous selenium (a-Se) - currently the only wide-bandgap photoconductor used commercially in the direct conversion detectors for low x-ray energy clinical applications (i.e., in x-ray mammography). In comparison to a-Se, a-PbO structural stability is much higher: the first a-PbO layers deposited two years ago and stored in air did not degrade and do not show any changes in both Raman and XRD spectra. This is an advantage over a-Se, since the later requires special doping for stabilization against recrystallization at ambient conditions, while un-stabilized pure a-Se structurally degrades in approximately one month after the deposition [29,30].

Perfect structural uniformity and stoichiometry of a-PbO layers is evident from the XPS analysis performed at different depths below the layer surface. In contrast, poly-PbO is over-oxidized at the surface while showing a certain deficit of oxygen in a bulk (as this is demonstrated by the presence of un-oxidized $\mathrm{Pb}$ in XPS spectra shown in Fig. 5 (a)). Oxygen deficiency is known to be a common problem of all metal oxides, since oxygen molecules are electrically neutral and thus do not react deliberately with other elements. Therefore, in order to promote incorporation of oxygen in the growing film, this charge neutrality must be distorted. Here, utilization of an ion source was a natural way of solving the oxygen deficiency problem. Indeed, the ion source ionizes oxygen, 
converting it to a charged state and thus making it more reactive. As a result of such an ion assisted deposition a stoichiometric $\mathrm{PbO}$ layers were received for the first time ever (see Fig. 5 (b)).

Overall, optimization of the ion assisted thermal deposition process allows one to grow amorphous $\mathrm{PbO}$ layers a new polymorphic configuration free of structural imperfections inherent to more common polycrystalline $\mathrm{PbO}$. The improvement includes higher film density and much better uniformity, stoichiometry and stability against structural changes. The amorphous $\mathrm{PbO}$ deposition process is compatible with large area $\mathrm{x}$-ray detector technologies. In such, an amorphous $\mathrm{PbO}$ x-ray-to-charge transducer should be considered as an alternative to a-Se for x-ray medical detectors in the diagnostic energy range where a-Se is suboptimal due to its low atomic number. Although comprehensive research on a-PbO electronic and photoconductive properties is needed, the reported results are very promising to extend the advances of direct conversion x-ray medical imaging over a variety of imaging procedures including radiography, fluoroscopy, pediatric imaging, etc. In addition, new a-PbO can also be considered for applications in large area photovoltaic devices [31], solar cell [32] and light emitting diodes [33], where electronic properties of $\mathrm{PbO}$ were shown to significantly improve the device performance.

\section{Conclusion}

We propose advanced deposition process for $\mathrm{PbO}$ that allows one to obtain a new polymorphic form of this material - amorphous lead oxide, as confirmed with Raman and XRD spectroscopies. In contrast to poly-PbO, a$\mathrm{PbO}$ samples are stable at ambient environment and perfectly stoichiometric. In addition, SEM indicates that new a$\mathrm{PbO}$ layers have bulk packing density, which offers high X-ray stopping power. Proposed advances in the deposition technology are easy to implement and hold the promise to utilize the full potential of $\mathrm{PbO}$ in medical imaging X-ray detectors as well as many other applications.

\section{Acknowledgement}

The authors are thankful to Dr. Matthias Simon, Philips Research Labs and Prof. Kai Wang, Joint Institute of Engineering at Carnegie Mellon University for help in optimization of the $\mathrm{PbO}$ growth process. The authors gratefully acknowledge the financial support from the National Science and Engineering Research Council (NSERC), Canadian Institute for Health Research (CIHR) and Ontario Research Fund-Research Excellence (ORFRE) program. Also, this publication is supported by the GINOP-2.3.2-15-2016-00041 project. The project is co- 
financed by the European Union and the European Regional Development Fund. Support by the TAMOP 4.2.2.A11/1/KONV-2012-0036 project, which is co-financed by the European Union and European Social Fund, is also acknowledged.

Conflict of Interest: The authors declare that they have no conflict of interest.

\section{References}

[1] Simon M, Ford RA, Franklin AR et al (2005) Analysis of lead oxide ( $\mathrm{PbO}$ ) layers for direct conversion x-ray detection. IEEE Trans Nucl Sci 52:2035-2040. doi: 10.1109/NSSMIC.2004.1466833

[2] Rowlands JA, Yorkstone J (2000) Flat panel detectors for digital radiography. In: Beutel J, Kundel HL, Van Metter RL (eds) Handbook of medical imaging, vol.1. SPIE Press, Bellingham pp 223-328

[3] Zentai G (2009) Photoconductor-based (direct) large-area x-ray imagers. JSID 17: 543-550. doi:10.1889/JSID17.6.543

[4] Macleod HA (2010) Factors affecting layer and coating properties. In: Brown RGW, Pike ER (eds) Thin film optical filters, 4th edn. CRC Press, Boca Raton, pp 569-584

[5] Anders A (2010) A structure zone diagram including plasma-based deposition and ion etching. Thin Solid Films 518:4087-4090. doi:10.1016/j.tsf.2009.10.145

[6] Semeniuk O, Juska G, Oelerich JO et al (2016) Charge transport mechanism in lead oxide revealed by CELIV technique. Sci Rep 6:33359. doi:10.1038/srep33359

[7] Wiechert DU, Grabowski SP, Simon M (2005) Raman spectroscopic investigation of evaporated PbO layers. Thin Solid Films 484:73-82. doi:10.1016/j.tsf.2005.02.010

[8] Bigelow JE, Haq KE (1962) Significance of fatigue in lead oxide vidicon target. J Appl Phys 33:2980-2982. doi: $10.1063 / 1.1728546$

[9] Hwang OH, Kim SS, Suh JH et al (2011) Effect of thermal annealing of lead oxide film. Nucl Instrum Methods Phys Res A 633:S69-S71. doi: 10.1016/j.nima.2010.06.125

[10] Scanlon DO, Kehoe AB, Watson GW (2011) Nature of the band gap and origin of the conductivity of $\mathrm{PbO}_{2}$ revealed by theory and experiment. Phys Rev Lett 107:246402. doi:10.1103/PhysRevLett.107.246402

[11] Berashevich J, Semeniuk O, Rubel O et al (2013) Lead monoxide $\alpha$-PbO: electronic properties and point defect formation. J Phys: Condens Matter 25:075803. doi:10.1088/0953-8984/25/7/075803

[12] Zhitomirsky I, Gal-Or L, Kohn A et al (1995) Electrochemical preparation of PbO films. J Mater Sci Lett 14:807-810. doi:10.1007/BF00278136

[13] Anders A (2005) Plasma and ion sources in large area coating: A review. Surf Coat Technol 200:1893-1906. doi:10.1016/j.surfcoat.2005.08.018

[14] Krishna MG, Rao KN, Mohan S (1992) Optical properties of ion assisted deposited zirconia thin films. J Vac Sci Technol A 10:3451-3455. doi:10.1116/1.577801 
[15] Allen TH (1982) Properties of ion assisted deposited silica and titania films. SPIE Proc 0325:93-100. doi: $10.1117 / 12.933291$

[16] Jensen TR, Warren J, Johnson RL (2002) Ion-assisted deposition of moisture-stable hafnium oxide films for ultraviolet applications. Appl Opt 41:3205-3210. doi:10.1364/AO.41.003205

[17] Netterfield RP, Sainty WG, Martin PJ et al (1985) Properties of $\mathrm{CeO}_{2}$ thin films prepared by oxygen-ionassisted deposition. Appl Opt 24:2267-2272. doi:10.1364/AO.24.002267

[18] Wang K, Abbaszadeh S, Karim KS et al (2015) Reactive ion-assisted deposition of cerium oxide holeblocking contact for leakage-current suppression in an amorphous selenium multilayer structure. IEEE Sens J 15:3871-3876. doi:10.1109/JSEN.2015.2397953

[19] McNeil JR, Barron AC, Wilson SR et al (1984) Ion-assisted deposition of optical thin films: low energy vs high energy bombardment. Appl Opt 23:552-559. doi:10.1364/AO.23.000552

[20] Wang L, Yoon MH, Lu G et al (2006) High-performance transparent inorganic-organic hybrid thin-film ntype transistors. Nat Mater 5:893-900. doi:10.1038/nmat1755

[21] Tan M, Deng Y, Hao Y (2013) Enhanced thermoelectric properties and superlattice structure of a $\mathrm{Bi}_{2} \mathrm{Te}_{3} / \mathrm{ZrB}_{2}$ film prepared by ion-beam-assisted deposition. J Phys Chem C 117:20415-20420. doi:10.1021/jp4053133

[22] Tepavcevic S, Choi Y, Hanley L (2003) Surface polymerization by ion-assisted deposition for polythiophene film growth. J Am Chem Soc 125:2396-2397. doi: 10.1021/ja029851s

[23] Farhan MS, Zalnezhad E, Bushroa AR et al (2013) Electrical and optical properties of indium-tin oxide (ITO) films by ion-assisted deposition (IAD) at room temperature. IJPEM 14:1465-1469. doi:10.1007/s12541-013$0197-5$

[24] Zhang XW, Boyen HG, Deyneka N (2003) Epitaxy of cubic boron nitride on (001)-oriented diamond. Nat Mater 2:312 - 315. doi:10.1038/nmat870

[25] NIST X-ray Photoelectron Spectroscopy Database. https://srdata.nist.gov/xps/. Accessed 12 December 2016

[26] Vad K, Csik A, Langer GA (2009) Secondary neutral mass spectrometry - a powerful technique for quantitative elemental and depth profiling analyses of nanostructures. Spectrosc Eur 21:13-17. ISSN: 09660941

[27] Geiger JF, Kopnarski M, Oechsner H (1987) SNMS-analysis of insulators. Mikrochim Acta 91:497-506. doi:10.1007/BF01199524

[28] Oechsner H (1995) Secondary neutral mass spectrometry (SNMS)-recent methodical progress and applications to fundamental studies in particle/surface interaction. Int J Mass Spectrom Ion Proces 143:271282. doi:10.1016/0168-1176(94)04122-N

[29] Kabir M, Kasap S, Rowlands A J (2006) Photoconductors for x-ray image detectors. In: Kasap S, Capper P (eds) Springer Handbook of Electronic and Phtonic Materials. Springer, New York, pp. 1125-1126

[30] Grenet J, Larmagnac JP, Michon P (1980) Aging and crystallization of evaporated amorphous selenium films. Thin Solid Films 67:L17-L20. doi:10.1016/0040-6090(80)90309-0

[31] Droessler LM, Assender HE, Watt AAR (2012) Thermally deposited lead oxides for thin film photovoltaics. Mater Lett 71:51-53. doi:10.1016/j.matlet.2011.12.027 
[32] Zhang H, Ouyang J (2011) High-performance inverted polymer solar cells with lead monoxide-modified indium tin oxides as the cathode. Org Electron 12:1864-1871. doi:10.1016/j.orgel.2011.07.023

[33] Wang Q, Deng Z, Ma D (2009) Highly efficient inverted top-emitting organic light-emitting diodes using a lead monoxide electron injection layer. Opt Express 17:17269-17278. doi:10.1364/OE.17.017269 